\title{
TELEREHABILITATION: AN ADJUNCT SERVICE DELIVERY MODEL FOR EARLY INTERVENTION SERVICES
}

\author{
JANA CASON, DHS, OTR/L \\ SPALDING UNIVERSITY, LOUISVILLE, KY
}

\section{ABSTRACT}

Early Intervention (EI) services for children birth through two years of age are mandated by Part $\mathrm{C}$ of the Individuals with Disabilities Education Act (IDEA); however, personnel shortages, particularly in rural areas, limit access for children who qualify. Telerehabilitation has the potential to build capacity among caregivers and local providers as well as promote family-centered services through remote consultation. This article provides an overview of research related to telerehabilitation and early intervention services; discusses the feasibility of telerehabilitation within traditional El service delivery models; examines telecommunications technology associated with telerehabilitation; and provides hypothetical case examples designed to illustrate potential applications of telerehabilitation in early intervention.

Keywords: Telerehabilitation, early intervention services, Individuals with Disabilities Education Act (IDEA) Part C, infants, toddlers, children

\section{TELEREHABILITATION: AN ADJUNCT SERVICE DELIVERY MODEL FOR EARLY INTERVENTION SERVICES}

Early intervention (EI) services for children from birth through two years of age are designed to promote development and improve the quality of life of infants and toddlers who have been identified as having a disability or developmental delay. A primary goal of El services is to enhance the capacity of families to help their child learn, achieve developmental milestones, and participate in home and community activities. El services for children from birth through two years of age are mandated by Part $\mathrm{C}$ of the Individuals with Disabilities Education Act (IDEA); however, personnel shortages, particularly in rural areas, limit access for children who qualify. Telerehabilitation is a service delivery model that demonstrates the potential to deliver El services effectively and efficiently, thereby ameliorating the impact of provider shortages in underserved areas.

\section{BRIEF HISTORY OF LEGISLATION RELATED TO EARLY INTERVENTION SERVICES}

Part $\mathrm{H}$ of the Education of All Handicapped Children Act (EHA) Amendments of 1986 (Public Law 99-457) created incentives for states to provide El services (Bruder, 2010; Hanson \& Bruder, 2001). In 1990, the EHA was revised and renamed the Individuals with Disabilities Education Act (IDEA). Since that time, the IDEA has been amended and reauthorized by the United States Congress several times, most recently in 2004 . The IDEA mandates the provision of educationally related services to children birth through 21 years of age. Under the IDEA, Part C (formerly Part H of EHA), federal legislation encourages states to provide El services to children birth through three years of age who have been identified as having a disability or developmental delay. At a state's discretion, children at-risk for developing a significant developmental delay may also participate in early intervention programming (Scarborough, Hebbeler, \& Spiker, 2006). El services as mandated by IDEA Part C enhance the functional development of young children with disabilities by connecting families and caregivers with resources and supports. These services enhance the capacity of families to meet the needs of their child with a disability by capitalizing on learning experiences that occur in everyday routines and contexts (Workgroup on Principles and Practices in Natural Environments, 2007). Early intervention services are provided within a child's natural environment or "settings that are natural or normal for the child's age peers who have no disabilities" (Code of Federal Regulations - 34 CFR §303.18, 2011). 


\section{OVERVIEW OF CURRENT RESEARCH RELATED TO TELEREHABILITATION IN EI SERVICES}

Telerehabilitation is a delivery model that uses telecommunications technology to provide therapeutic services at a distance. A review of the literature located five articles associated with the use of telerehabilitation in El services for children birth through two years of age (Cason, 2009; Heimerl \& Rasch, 2009; Kelso, Fiechtl, Olsen, \& Rule, 2009; Vismara, Young, Stahmer, Griffith, \& Rogers, 2009; Wakeford, 2002). Cason (2009) investigated the use of telerehabilitation to provide early intervention occupational therapy to two families living in a rural community with limited access to services. The study demonstrated that telerehabilitation is a feasible service delivery model that has the potential to improve access and reduce the costs associated with delivery of early intervention services in rural communities. Heimerl and Rasch (2009) conducted 224 telerehabilitation encounters between 2004 and 2006 and concluded that "telehealth services are not meant to replace face-to-face services, but when in-person services are not feasible, they provide a viable alternative" (p. 3). Kelso, Fiechtl, Olsen, \& Rule (2009) used two-way interactive telecommunications technology over the Internet to supplement face-toface early intervention services for four families living in a remote area. A high level of satisfaction among the interventionists and families and a cost-benefit analysis resulted in the authors concluding that telerehabilitation "appears both feasible and beneficial" and that "early intervention programs could use this delivery method to more adequately serve young children with disabilities either instead of or in addition to making traditional home visits" (p. 339). Lastly, Vismara, Young, Stahmer, Griffith, and Rogers (2009) investigated the efficacy of telehealth technology as a training medium for early intervention providers. This study demonstrated that telehealth technology was as effective as face-to-face instruction. These studies provide evidence for the feasibility of telerehabilitation as a delivery model in early intervention, particularly in areas where provider shortages limit access to services.

While the research related to telerehabilitation in El is limited, there is a substantial body of literature on the use of telemedicine and telehealth in pediatrics. Many of the findings reported in this body of literature appear to generalize to telerehabilitation. For example, when describing the results of an 8-year telemedicine project designed to evaluate the efficacy of team-to-team interdisciplinary telemedicine evaluations for children with special needs in rural lowa, Harper (2006) stated "teamto-team consultation permits comprehensive parent and professional dialogue, professionally guided evaluation procedures, real-time discussion of evaluation results, treatment recommendations, and coordination of care" (p. 12). In addition to benefits associated with real-time remote consultation among team members, this and other research have found a high level of satisfaction among participants, improved access to services, and significant economic savings (Baharav \& Reiser, 2010; Cason, 2009; Harper, 2006; Heimerl \& Rasch, 2009; Karp et al, 2000; Kelso et al, 2009; Robinson, Seale, Tiernan, \& Berg, 2003). Use of telerehabilitation in El services has the potential to connect team members remotely to discuss evaluation results, treatment recommendations, and coordinate care as well as facilitate co-treatments with specialists and local therapists and provide access to specific disciplines not available within a local community.

\section{TELEREHABILITATION AS AN ALTERNATIVE} Service Delivery Model For EI Services

Currently, all fifty states, the District of Columbia, and five jurisdictions (American Samoa, Guam, Northern Mariana Islands, Palau, and Republic of the Marshall Islands) provide El services under IDEA Part C (NECTAC, 2010). States and participating jurisdictions report annually to the Office of Special Education Programs (OSEP) within the US Department of Education. This federal agency oversees implementation of the IDEA Part C 14 indicators through State Performance Plans (SPP) and Annual Performance Reports. Telerehabilitation has the potential to improve state performance on many of the reported indicators. For example, Indicator 1 relates to the provision of El services in a timely manner. Many states report noncompliance with this indicator due to personnel shortages resulting in the inability to serve children in a timely manner, particularly in rural areas. According to the National Early Childhood Technical Assistance Center (NECTAC) 2010 annual report:

"The most frequently cited reason for slippage or lack of progress in providing services in a timely manner [Indicator 1] continued to be personnel shortages. Particularly acute was the lack of therapists in rural areas of the State. Staff vacancies, inability to pay competitive salaries, and heavy caseloads were cited as major issues in all areas of the country; however, some States are devising methods to increase funding. Additional reasons for lack of progress were procedural or funding issues including delays in billing and insurance authorization or the inability to pay or reimburse mileage for the long distances that providers need to travel to reach some families. Because of these constraints, States reported it was difficult to find providers willing to provide services in natural environments" (p. 6).

Telerehabilitation may improve state performance on this and other OSEP indicators by providing remote access to qualified personnel and by building capacity of local providers through consultation with remote experts. Table 1 outlines where telerehabilitation may prove beneficial in improving states' performance on selected OSEP indicators. 
Table 1

\section{Selected OSEP Indicators and Potential Applications of Telerehabilitation}

\section{Indicator 1: Timely Receipt of Services}

Percent of infants and toddlers with Individualized Family Service Plans (IFSPs) who receive the early intervention services on their IFSPs in a timely manner.

\section{Potential Benefit of Telerehabilitation}

Improve timely receipt of services by remotely increasing access to providers/services not available within a local community.

\section{Indicator 2: Settings}

Percent of infants and toddlers with IFSPs who primarily receive early intervention services in the home or community-based settings.

\section{Potential Benefit of Telerehabilitation}

Maintain provision of services within the home or community-based settings by:

- using technology within the home or community-based settings when available.

- supplying local providers with mobile videoconferencing technologies to connect with remote providers from the home or community-based settings.

- utilizing existing infrastructure (telehealth networks) to tap into the expertise of a provider not available within a local community and then implement the strategies and recommendations within the home or community-based settings.

\section{Indicator 3: Infant \& Toddler Outcomes}

Percent of infants and toddlers with IFSPs who demonstrate improved: (a) Positive social-emotional skills (including social relationships); (b) Acquisition and use of knowledge and skills (including early language/communication); and (c) Use of appropriate behaviors to meet their needs.

\section{Potential Benefit of Telerehabilitation}

Improve infant and toddler outcomes by using telerehabilitation to:

- access providers/services not available within a local community.

- consult with parents and caregivers to enhance skill development during naturally occurring routines.

- conduct professional development activities for providers.

- provide training for child outcomes data collection and reporting.

\section{Indicator 4: Family Outcomes}

Percent of families participating in Part $\mathrm{C}$ who report that early intervention services have helped the family: (a) Know their rights; (b) Effectively communicate their children's needs, and (c) Help their children develop and learn.

\section{Potential Benefit of Telerehabilitation}

Improve family outcomes by using telerehabilitation to conduct ongoing provider training on effective consultative and coaching strategies so that families' experiences in El leads to the desired outcomes.

\section{Indicator 5: Child Find Birth to One}

Percent of infants and toddlers birth to one with IFSPs compared to national data.

\section{Indicator 6: Child Find Birth to Three}

Percent of infants and toddlers birth to 3 with IFSPs compared to national data.

\section{Potential Benefit of Telerehabilitation}

Promote Child Find efforts using telerehabilitation to:

- facilitate development and implementation of public awareness activities and materials.

- engage in outreach activities with physicians and referring agencies.

- connect experts to explore best practices related to evaluation and assessment of children birth to 3 years.

- provide immediate access to interpreters when families call with a referral through a contracted "language line" service.

\section{Indicator 7: 45-day Timeline}

Percentage of eligible infants and toddlers with IFSPs for whom an evaluation and assessment and an initial IFSP meeting were conducted within Part C's 45-day timeline.

\section{Potential Benefit of Telerehabilitation}

Improve timely receipt of services by remotely increasing access to providers/services not available within a local community. Utilize telerehabilitation to overcome challenges with personnel shortages (e.g. evaluators, service coordinators, developmental specialists, therapists), severe weather prohibiting travel, and access to interpreters.

\section{Indicator 8 (c): Transition}

Percent of all children exiting Part $\mathrm{C}$ who received timely transition planning to support the child's transition to preschool and other appropriate community services by their third birthday, including transition conference.

\section{Potential Benefit of Telerehabilitation}

Improve timely transition conferences by fostering timely meetings through remote access to conferences for service coordinators, other service providers and families. 


\section{APPLICABILITY OF TELEREHABILITATION Within TRADITIONAL EI SERVICE DELIVERY MODELS}

Key features of El services required by IDEA Part $C$ include the utilization of family-centered services and implementation of services within a child's natural environment (IDEA Part C, 34CFR 303.12(b); 34CFR303.18; Section 632(4) (6)). Family-centered services are characterized by the philosophy that a family is a constant in a child's life, and that services that support a family also benefit their child with a disability or developmental delay. To this end, services should reflect the needs and priorities identified by the family, and professionals should work with families as partners towards shared goals (Trute \& Heiber, 2007).

In addition to El services being family-centered, services must also be provided within a child's natural environment such as the home or community settings where developing peers typically spend time. When necessary, there are provisions for services to occur in settings other than a child's natural environment (e.g. clinic, rehabilitation hospital); however, these services must be justified and periodically re-evaluated and discussed by the child's El service team.

\section{Direct Versus Consultative Services}

Though there are federally mandated key features of El services as outlined above, El service delivery models vary widely by state and may include services that are direct or consultative in nature. Direct services focus interventions toward the child receiving services, and frequently utilize hands-on techniques with the child to promote skill development. In contrast, consultative services focus interventions toward the child's caregivers to transfer knowledge and skills that will support the child's development. A common misconception is that consultative services do not include any direct handson interventions; however, this is not the case. Using the technique of coaching within a consultative model, the early interventionist incorporates both "hands-on" and "hands-off" intervention and uses direct service for assessment and modeling of therapeutic techniques and instructional methods to promote the child's skill development (Rush, Shelden, \& Hanft, 2003). Consultative services are used to enhance a caregiver's capacity to embed learning opportunities throughout a child's naturally occurring routines to promote skill development and generalization of skills across environments (Dinnebeil, Pretti-Frontczak, \& Mclnerney, 2009).

Telerehabilitation aligns with the consultative service delivery model and may be used to connect specialists or disciplines unavailable within a local community for a child's El service team. Through remote consultation, telerehabilitation has the potential to build capacity among caregivers and local providers and promote family-centered services. Using telerehabilitation, team members can work together to identify learning opportunities within a child's natural environments, teach therapeutic techniques to embed within daily routines, collaboratively problem-solve, coordinate care, and identify community and family resources.

\section{EI TEAMING MODELS: MULTIDISCIPLINARY, INTERDISCIPLINARY, AND TRANSDICIPLINARY}

Telerehabilitation also aligns with El teaming models, the philosophical and organizational structure that guides the interactions of El team members. Multidisciplinary, interdisciplinary, and transdiciplinary models are the primary teaming models used in El services. Key distinctions between the models are evidenced by assessment process, interaction styles and frequency, and child outcomes (see Table 2). Telerehabilitation can be utilized within the interdisciplinary and transdiciplinary team models to facilitate assessment, communication, collaboration, coaching, role-release, and mobilization of resources and family supports. 
Table 2

\section{El Teaming Models}

\author{
Assessment process \\ Multidisciplinary \\ - Individual assessment by each provider \\ - Discipline-specific assessment \\ Interdisciplinary \\ - Individual or arena assessment (team) \\ - Discipline-specific assessment or integrated \\ assessment in multiple domains \\ Transdisciplinary \\ - Arena assessment \\ - Integrated assessment in multiple domains \\ - Authentic, functional assessment with multiple \\ observations across settings and data from multiple \\ sources.
}

\section{Interaction styles and frequency}

\section{Multidisciplinary}

- Limited communication between providers

-Well-defined discipline specific role

\section{Interdisciplinary}

- More frequent formal and informal communication between providers

- Utilization of "co-treatments" for collaboration among providers

- Relatively well-defined discipline-specific role

Transdisciplinary

- Frequent formal and informal communication between providers

- Utilization of "co-treatments" for the purpose of collaboration and "role-releasing" of skills to other providers.

- Less-defined discipline specific role

- May designate a "primary service provider" to take lead in implementation of plan

\section{Child outcomes}

\section{Multidisciplinary}

- Each provider works with family/child on disciplinespecific outcomes

Interdisciplinary

- Providers work towards shared outcomes

- Child outcomes are functional and integrated across domains.

Transdisciplinary

- Providers work towards shared outcomes

- Child outcomes are functional and integrated across domains.

\section{TECHNOLOGY InFRASTRUCTURE}

The plethora of commercial and consumer videoconferencing products create many technology options for telerehabilitation within El services (see Table 3). There are several technology options for videoconferencing within the home environment including Voice over Internet Protocol (VoIP) services, mobile videoconferencing systems, Plain Old Telephone Service (POTS) videoconferencing, and high definition television (HDTV) technologies. VolP service uses a computer, special VolP phone, or traditional phone with adapter to convert voice into a digital signal that travels over the Internet (Federal Communications Commission, 2010). Integrated with video software, VoIP provides a mechanism for Internet-based videoconferencing. Similarly, mobile videoconferencing uses a cell phone with videoconferencing capabilities to transmit audio and video over a Wi-Fi or cellular network. Cell phones may also be tethered to a laptop computer and serve as a modem for Internet-based videoconferencing. Plain Old Telephone Service (POTS) videoconferencing primarily uses an analog telephone line or landline to support audio and video transmission through a videophone or specialized equipment connected to a television. High definition television (HDTV) videoconferencing requires a high definition television, console, HD camera, remote control and high speed broadband connection at both locations. Unlike the technologies described above and marketed for consumer use, state designated telehealth networks utilize high-end videoconferencing technologies (e.g., Polycom, Tandberg) and fiberoptic telephone lines (e.g., T1 lines) or high-speed Internet to connect sites throughout the state.

Advantages of Voice over Internet Protocol (VolP), mobile, POTS, and consumer HDTV technologies include service provision within a child's natural environment with no travel necessary for families, minimal infrastructure requirements, and lower costs for equipment and connectivity (e.g., residential service plan). Disadvantages may include privacy, security, and confidentiality risks, lack of infrastructure (e.g. limited access to highspeed Internet/Broadband; inadequate bandwidth for connectivity within rural communities), diminished sound/image quality, and technological challenges associated with end-user experience and expertise with videoconferencing technology. Advantages of leveraging established telehealth networks with commercial videoconferencing systems include the likelihood of reliable high-quality videoconferencing sessions that meet security and privacy provisions (outlined in the Health Insurance Portability and Accountability Act (HIPAA) and the Family Education Rights and Privacy Act (FERPA)) and increased telehealth network efficiency as a result of minimal equipment down-time. Additionally, clinical use of established telehealth networks circumvents challenges associated with infrastructure limitations related to rural 
communities, socio-economic barriers, and limited end-user experience and expertise with technology. Disadvantages may include costs associated with the need for increased staffing at telehealth sites, travel requirements for families and providers, and a portion of a child's El service occurring outside of the natural environment. Though telehealth sites are not considered part of a child's natural environment, recommendations provided through telerehabilitation by specialists and discipline-specific providers not available within the local community can be implemented by families and providers within a child's natural environment. In this way, leveraging established telehealth networks results in the majority of El services occurring within a child's natural environment.

\section{Table 3}

\section{Technology Options for Telerehabilitation within El Services}

\section{Technology}

Voice Over Internet Protocol (VoIP Technologies)

\section{Example}

Skype, Oovoo, iChat Google Voice and Video Chat, Tandberg

Movi

\section{Technology}

Mobile videoconferencing

\section{Example}

Cell phone as modem tethered to computer, Apple IPhone

\section{Technology}

Consumer HDTV videoconferencing

Example
Cisco Umi; Galaxy Tabs (Samsung/ Polycom)

\section{Technology}

Plain Old Telephone Service (POTS)

Videoconferencing

\section{Example}

NextLINK videophone

\section{Technology}

Telehealth network with commercial videoconferencing system

\section{Example}

Polycom, Tandberg

\section{Considerations}

- Confidentiality ${ }^{1}$ (security, privacy)

- Integrity (information protected from changes by unauthorized users)

- Availability ${ }^{1}$ (information, services)

- Cost/benefit ratio

-Socio-economical considerations

- Leveraging existing infrastructure (equipment/personnel)

- Technology connection requirements (e.g. Broadband, T1 line)

- Sound and image quality

- Equipment accessibility

- Provider/end-user comfort, experience, and expertise with technology

\footnotetext{
${ }^{1}$ For an in-depth discussion of VolP risk analysis see Watzlaf, V., \&, Moeini, S., \& Firouzan, P. (2010). VolP for telerehabilitation: A risk analysis for privacy, security, and HIPAA compliance. International Journal of Telerehabilitation, 2(2), 3-14. doi: 10.5195/ijt.2010.6056
} 


\section{CASE EXAMPLES}

The following are hypothetical case examples designed to demonstrate potential applications of telerehabilitation in El services for children from birth through two years of age using VolP technology, mobile videoconferencing, consumer HDTV videoconferencing, and a state telehealth network.

\section{CASE EXAMPLE \#1: VOIP TECHNOLOGY}

Emily, a 16-month old child, received service coordination, speech therapy, and occupational therapy since qualifying for El services at the age of 6-months when severe feeding and sensory issues were identified. As time progressed, a language delay also emerged. Emily's speech-language pathologist traveled 2-hours roundtrip to see Emily and was only in Emily's community two times each month. When a scheduled visit was cancelled due to illness, weather, or another event, Emily was seen by the speech-language pathologist once in that particular month. Due to progress in language acquisition, the El service team agreed that Emily would benefit from speech therapy on a weekly basis, however, in-person visits at that frequency was not possible due to commuting distance and availability of the speech-language pathologist. The team decided to supplement the in-person speech therapy sessions with remote speech therapy sessions using Internet-based videoconferencing (Skype). After providing informed consent, Emily's caregiver connected from home with the speech-language pathologist at a scheduled time through the Internet using a computer and web camera. This service delivery model was used to supplement the inperson speech therapy visits and resulted in Emily making rapid progress.

\section{CASE EXAMPLE \#2: MOBILE VIDEOCONFER- ENCING (CELl PHONE AS MODEM TETHERED TO LAPTOP COMPUTER)}

Katelyn, a two-year old child, qualified for El services to address delays in motor skills, adaptive (self-help) skills, and communication. Additionally, concerns related to Katelyn's ability to process sensory information were identified as having an impact on her ability to self-regulate and participate in daily routines. The El service team consisted of a service coordinator, physical therapist, and speech-language pathologist. Occupational therapy was recommended, however, an occupational therapist was not available within Katelyn's community. After obtaining appropriate informed consent, the service coordinator used a cell phone as a modem tethered to a laptop computer to connect an occupational therapist with Katelyn's caregiver and El service providers remotely using videoconferencing software. During this meeting, the occupational therapist collaborated with the El service team, discussed concerns, and identified therapeutic techniques and strategies that could be embedded by Katelyn's caregiver and service providers into Katelyn's naturally occurring routines (mealtime, playtime, bath time, etc.). The occupational therapist offered recommendations to promote Katelyn's development within the motor, adaptive, and sensory areas and through technology provided a professional perspective that would otherwise have been unavailable. During subsequent virtual meetings, the occupational therapist was able to recommend and virtually demonstrate new strategies and techniques for Katelyn's caregiver and service providers to implement with Katelyn. As a result of mobile videoconferencing, Katelyn was able to benefit from the expertise of an occupational therapist with specialized knowledge and skills in the area of pediatric therapy and sensory processing. Additionally, Katelyn's local service providers benefitted from mobile videoconferencing with the occupational therapist, as their knowledge and skills were enhanced through remote consultation.

\section{CASE EXAMPLE \#3: CONSUMER HDTV VIDEOCONFERENCING (CISCO UMI)}

Ray, a 16-month old child with mild cerebral palsy, received El services including service coordination, developmental intervention, and speech therapy. Though the speech-language pathologist had some experience working with children with oral-motor and feeding difficulties, the therapist felt that a consultation with an expert in the area of feeding and swallowing would enhance Ray's progress. Ray's family and speech-language pathologist traveled to a rehabilitation hospital one-hour away to consult with a feeding expert and complete an intensive feeding and swallowing assessment. With the caregiver's informed consent, the El service team used the Cisco Umi HDTV videoconferencing system to connect with the feeding and swallowing expert on a monthly basis for follow-up consultation. This device was chosen for its visual and sound quality and the ability to pan and zoom the camera to observe Ray close-up as well as at a distance within his environment. During these virtual consultations, Ray's caregiver and speech-language pathologist discussed Ray's progress and any new concerns that had emerged with the feeding expert. The expert observed Ray eating, drinking, and interacting with his caregiver and therapist. Based on the discussion and observations, the expert provided additional strategies and techniques related to oral-motor skills and feeding to advance Ray's skills. As a result, Ray made excellent progress and the speechlanguage pathologist gained additional pediatric feeding 
and swallowing expertise through virtual consultations and a mentoring relationship with the remote feeding and swallowing expert.

\section{CASE EXAMPle \#4: STATE TELEHEALTH NETWORK}

Michael, a 29-month old child, was referred for an intensive level interdisciplinary evaluation at a Child Evaluation Center due to concerns and lack of progress noted by his El service team. Because Michael lived in a remote part of the state and was several hours away from the Child Evaluation Center (making travel for the family difficult), the Center and El service team decided to complete the interdisciplinary evaluation remotely using the State's Telehealth Network Videoconferencing system. Michael and his family traveled 15 miles from their home to the local health department, one of a thousand sites comprised of health departments, primary schools, secondary schools, public libraries, and universities within the state designated as Telehealth Network origination sites. After completing the appropriate informed consent, the El service team, Michael, and his parents connected with the interdisciplinary team at the Child Evaluation Center that consisted of a developmental pediatrician, speech-language pathologist, occupational therapist, and physical therapist. The Child Evaluation Center team completed interview-based assessments with Michael's parents and El service providers, remotely observed Michael engaged in specified tasks, and supplemented the assessment with information provided by the local El service providers including information on muscle tone and vital statistics. Through this process, the interdisciplinary team at the Child Evaluation Center diagnosed Michael with autism and provided Michael's parents and local El service team with recommendations to mobilize supports and resources within his community.

\section{DISCUSSION}

Telerehabilitation shows promise as an adjunct service delivery model for early intervention services for children birth through two years of age. By improving access to early intervention providers, states may find the utilization of telerehabilitation to be a positive strategy for improved performance on the Office of Special Education Program's Early Intervention Indicators reported by states annually. Time-sensitive indicators, including Indicator 1 (Timely-receipt of services) and Indicator 7 (45-day timeline), may improve most as a result of increased access to providers, though other indicators will also likely be positively impacted through the use of telerehabilitation as an adjunct service delivery model (see Table 1).
Local infrastructure, technology access and cost, provider and caregiver expertise and experiences with technology, and therapeutic needs of the child need to be considered when determining the best technology solution for telerehabilitation encounters. Videoconferencing technologies designed for personal and professional use are being increasingly marketed to the public. As a result, caregivers and providers are gaining awareness and knowledge of technology used for telerehabilitation. Increased awareness and personal use of videoconferencing technologies will likely lead to increased acceptance and willingness to explore these technologies for the delivery of El services.

Extensive training materials for caregivers and providers should be developed by states considering telerehabilitation as a service delivery model within their early intervention system. A repository that enables states to share training materials, resources, and competency guidelines and standards would prove cost-effective and will help states avoid "reinventing the wheel" as they seek ways to facilitate the use of telerehabilitation within their early intervention system.

Additionally, it is essential to establish provider competency standards designed to ensure that providers demonstrate adequate technical and therapeutic skills for the delivery of El services using telerehabilitation and are compliant with pertinent federal privacy, security, and early intervention laws and regulations.

\section{CONCLUSIONS}

Telerehabilitation has the potential to enhance early intervention service provision for children participating in IDEA Part $\mathrm{C}$ programming by enabling access to services unavailable within local communities and by complementing existing services. Telerehabilitation is not suggested to replace available face-to-face services, but rather to provide access to specific disciplines or specialized providers not available within a community. Using a consultative model, telerehabilitation can be used to facilitate assessment, communication, collaboration, coaching, role-release, and coordination of care. Even when telerehabilitation is provided in a setting other than a child's natural environment (e.g. established telehealth network site, clinic), recommendations, strategies, and therapeutic techniques can be implemented by a child's El service team within the natural environment. In this way, telerehabilitation complements face-to-face services and promotes family-centered services. Research is needed to determine the requisite skills and qualities of El providers using telerehabilitation, El assessments, interventions, strategies and techniques that can be effectively delivered through telerehabilitation, as well as optimal technologies for the delivery of El services using telerehabilitation. 


\section{REFERENCES}

1. Baharav, E. \& Reiser, C. (2010). Using telepractice in parent training in early autism. Telemedicine and e-Health, 16, 727-731.

2. Bruder, M. (2010). Early childhood intervention: $A$ promise to children and families for their future. Council for Exceptional Children, 76, 339-355.

3. Cason, J. (2009). A pilot telerehabilitation program: Delivering early intervention services to rural families. International Journal of Telerehabilitation, 1, 29-37.

4. Code of Federal Regulations. (2011). 34 CFR 303.18 - Natural environments. Retrieved from http://cfr.vlex. com/vid/303-natural-environments-19760129.

5. Dinnebeil, L., Pretti-Frontczak, K., \& Mclnerney, W. (2009). A consultative itinerant approach to service delivery: Consideration for the early childhood community. Language, Speech, and Hearing Services in Schools, 40, 435-445.

6. Federal Communications Commission. (2010). VoiceOver-Internet protocol. Retrieved from http://www.fcc. gov/voip/

7. Hanson, M., \& Bruder, M. (2001). Early intervention: Promises to keep. Infant and Young Children, 13(3), 47-58.

8. Harper, D. (2006). Telemedicine for children with disabilities. Children's Health Care, 35(1), 11-27.

9. Heimerl, S., \& Rasch, N. (2009). Delivering developmental occupational therapy consultation services through telehealth. Developmental Disabilities Special Interest Section Quarterly, 32(3), 1-4.

10. Karp, W., Grigsby, K., McSwiggan-Hardin, M., Pursley-Crotteau, S., Adams, L., Bell, W., Kanto, W. (2000). Use of telemedicine for children with special health care needs. Pediatrics, 105, 843-847.

11. Kelso, G., Fiechtl, B., Olsen, S., \& Rule, S. (2009). The feasibility of virtual home visits to provide early intervention: A pilot study. Infants \& Young Children, 22, 332-340.

12. National Early Childhood Technical Assistance Center (NECTAC). (2010). Part C SPP/APR 2010 Indicator Analyses - (FFY 2008). Retrieved from http://www. nectac.org/ pdfs/partc/part-c_sppapr_10.pdf

13. Robinson, S., Seale, D., Tiernan, K., \& Berg, B. (2003). Use of telemedicine to follow special needs children. Telemedicine and e-Health, 9, 57-61.

14. Rush, D., Shelden, M., \& Hanft, B. (2003). Coaching families and colleagues: A process for collaboration in natural settings. Infants \& Young Children, 16(1), 33-47.

15. Scarborough, A., Hebbeler, K., \& Spiker, D. (2006). Eligibility characteristics of infants and toddlers entering early intervention services in the United States. Journal of Policy and Practice in Intellectual Disabilities, 3, 57-64.

16. Trute, B., \& Hiebert-Murphy, D. (2007). The implications of "working alliance" for the measurement and evaluation of family-centered practice in childhood disability services. Infants \& Young Children, 20, 109-119.

17. Vismara, L., Young, G., Stahmer, A., Griffith, E., \& Rogers, S. (2009). Dissemination of evidence-based practice: Can we train therapists from a distance? Journal of Autism and Developmental Disorders, 39, 1636-1651.

18. Wakeford, L. (2002, November 25). Telehealth technology for children with special needs. OT Practice, 12-16.

19. Watzlaf, V., Moeini, S., \& Firouzan, P. (2010). VolP for telerehabilitation: A risk analysis for privacy, security, and HIPAA compliance. International Journal of Telerehabilitation, 2(2), 3-14. doi: 10.5195/ijt.2010.6056

20. Workgroup on Principles and Practices in Natural Environments. (2007, November). Mission and principles for providing services in natural environments. OSEP TA Community of Practice-Part C Settings. Retrieved from http://www.nectac.org/ topics/families/families.asp 


\section{ADDITIONAL RESOURCES}

1. American Occupational Therapy Association's Position Paper on Telerehabilitation, http://media.americantelemed.org/ICOT/AOTA\%20 telereh05.pdf

2. American Physical Therapy Association's Position on Telehealth, http://www.apta.org/AM/Template.cfm?Section=Home \&CONTENTID=67435\&TEMPLATE=/CM/ContentDisplay $\mathrm{cfm}$

3. American Speech-Language-Hearing Association's Position Statement on Telepractice http://www.asha.org/ docs/html/PS2005-00116.html

4. American Telemedicine Association, http://www. americantelemed.org

5. American Telemedicine Association's A Blueprint for Telerehabilitation Guidelines, http://www.americantelemed.org/files/public/standards/ ATA\%20Telerehab\%20Guidelines\%20v1\%20(2).pdf; also available at http://telerehab.pitt.edu/ojs/index.php/ Telerehab/article/view/6063/6293

6. Center for Telehealth and E-Health Law (CTel), http:// www.telehealthlawcenter.org/

7. International Journal of Telerehabilitation, http:// telerehab.pitt.edu/ojs/index.php/telerehab

8. Journal of Telemedicine and Telecare, http://jtt. rsmjournals.com/

9. Rehabilitation Engineering Research Center for Telerehabilitation, http://www.rerctr.pitt.edu Telemedicine and e-Health, www.liebertpub.com/TMJ

\section{AUTHOR ACKNOWLEDGEMENTS}

The author would like to thank several people who graciously provided expert and editorial review of this paper: Joicey Hurth, Associate Director, Technical Assistance for The National Early Childhood Technical Assistance Center (NECTAC); Lesly S. Wilson, Ph.D., OTR/L, Research Assistant Professor, University of South Carolina Team for Early Childhood Solutions (TECS); Tammy Richmond, MS, OTR/L, Chief Operating Officer of Ultimate Rehab and Sr. Clinical Analyst with THE FOTO GROUP, Inc; Sam Meyer, Writing Specialist, Spalding University Academic Resource Center; and Peggy Green. Additionally, the author would like to express gratitude to the Foundation for a Healthy Kentucky whose community grant provided the seed money that initiated the author's journey in telerehabilitation research, and to the following individuals for their mentorship and support: Rob Sprang, Director of the Kentucky Telehealth Network; Laura Strickland, Ed.D., OTR/L, Chair of the Auerbach School of Occupational Therapy at Spalding University, and L. Randy Strickland, Ed.D., OTR/L, Provost, Spalding University, Louisville, KY. 
INTERNATIONAL JOURNAL OF TELEREHABILITATION · TELEREHAB.PITT.EDU $\coprod$ 
